\title{
De-bugging the system: could antibiotics improve liver transplant outcomes?
}

\author{
Jonathan S. Bromberg, ${ }^{1,2,3}$ Joseph R. Scalea, ${ }^{1,2,3}$ and Emmanuel F. Mongodin ${ }^{1,2,4}$ \\ 'Department of Surgery, ${ }^{2}$ Department of Microbiology and Immunology, ${ }^{3}$ Center for Vascular and Inflammatory Diseases, and ${ }^{4}$ Institute for Cenome Sciences, University of Maryland School of Medicine, \\ Baltimore, Maryland, USA.
}

\begin{abstract}
Organ transplantation is now a preferred treatment for end-stage organ failure. Among the challenges for ensuring excellent clinical outcomes for transplant recipients is good initial allograft function at the time of organ implantation. This is determined in part by the functional status of the donor and donor organ, functional status of the recipient, and conduct of the operative procedure. Despite optimization of these variables, organ transplantation is still often plagued by substantial initial dysfunction, variably referred to as slow or delayed graft function, or in the most extreme cases, primary graft nonfunction necessitating urgent regrafting. In this issue of the $\mathrm{JCl}$, Nakamura, Kageyama, Ito, Hirao, and colleagues investigate a potential role for the recipient's microbiome in determining graft function after liver transplantation and demonstrate the benefits of antibiotic pretreatment in both a mouse model and in human patients.
\end{abstract}

\section{Effect of the recipient's gut microbiome on liver transplantation}

Although many factors have been ascribed to poor initial graft function, ischemiareperfusion injury (IRI) remains prominent among them (1). The causes of allograft IRI have usually been ascribed to poor donor organ quality, prolonged cold ischemia time, and suboptimal preservation technologies (2). Yet, even in optimal circumstances, IRI still occurs, so there must be other contributing events. In this issue, a team from the laboratory of Jerzy KupiecWeglinksi, who have been leaders in the field of hepatic IRI for many years, assessed the potential role of the recipient's gut microbiota as a driver of the initial IRI observed after liver transplantation (3).

The researchers focused on the gut microbiota because of its established role in the pathophysiology of liver diseases, including nonalcoholic fatty liver disease
(NAFLD) and nonalcoholic steatohepatitis (NASH), and their progression to cirrhosis, end-stage liver disease, and hepatocellular carcinoma (4). It is also now apparent that the microbiome can be a causal agent in numerous metabolic, vascular, inflammatory, and neurohormonal events, with far-reaching consequences for disease pathophysiology (5). To evaluate the role of the gut microbiome in liver transplantation outcomes, the team first assessed the influence of oral antibiotic treatment on IRI severity in a clinically relevant murine allogeneic liver transplant model, with prolonged ex vivo cold storage at $4^{\circ} \mathrm{C}$ for 18 hours, which mimics extremely marginal human liver grafts. Recipient mice were treated for 10 days with oral amoxicillin prior to transplantation. Antibiotic treatment reduced many early measures of hepatic IRI, including elevated transaminase, elevated serum and intrahepatic cytokine levels, hepato-

Delated Article: p. 3420

Conflict of interest: The authors have declared that no conflict of interest exists.

Copyright: @ 2019, American Society for Clinical Investigation.

Reference information: / Clin Invest. 2019;129(8):3054-3057. https://doi.org/10.1172/JCI130314.

cellular apoptosis, and hepatic myeloid cell infiltration (3).

\section{Identifying a molecular mechanism}

On the basis of their prior work elucidating the interrelated cellular and biochemical pathways that regulate metabolism during IRI (6-8), Nakamura, Kageyama, Ito, Hirao, and colleagues investigated the roles of endoplasmic reticulum (ER) stress, autophagy, prostaglandin E2 (PGE2), PGE2 receptor 4 (EP4), and cyclooxygenase 2 (COX2) in their model (3). They found that antibiotic therapy increased Cox 2 gene expression in the intestine and PGE2 metabolites in the portal venous circulation (Figure 1), suggesting that microbiotaintestinal crosstalk may regulate COX2 expression and PGE2 production. Antibiotic treatment also increased hepatic EP4 expression and suppressed ER stress. In complementary in vitro experiments, the authors showed that treating hepatocytes with PGE2 stimulated EP4, which suppressed ER stress and induced autophagy. Conversely, EP4 antagonism had the opposite effect, increasing in vivo IRI measures of transaminitis, apoptosis, and hepatic and systemic inflammatory infiltrates and cytokines. Taken together, these experiments reveal a remarkable concatenation of events leading from microbiota-intestinal communication through several receptor-ligand events that control basic cellular functions and determine the development of IRI after transplantation (Figure 1).

\section{Translating a mouse model to human patients}

The limitations of this approach are that the murine recipients did not have cirrhosis, and longer-term consequences on hepatic function or recipient survival were not assessed. Thus, though the authors demonstrated an immediate effect of antibiotic therapy on IRI, this result might not translate into durable outcomes in 


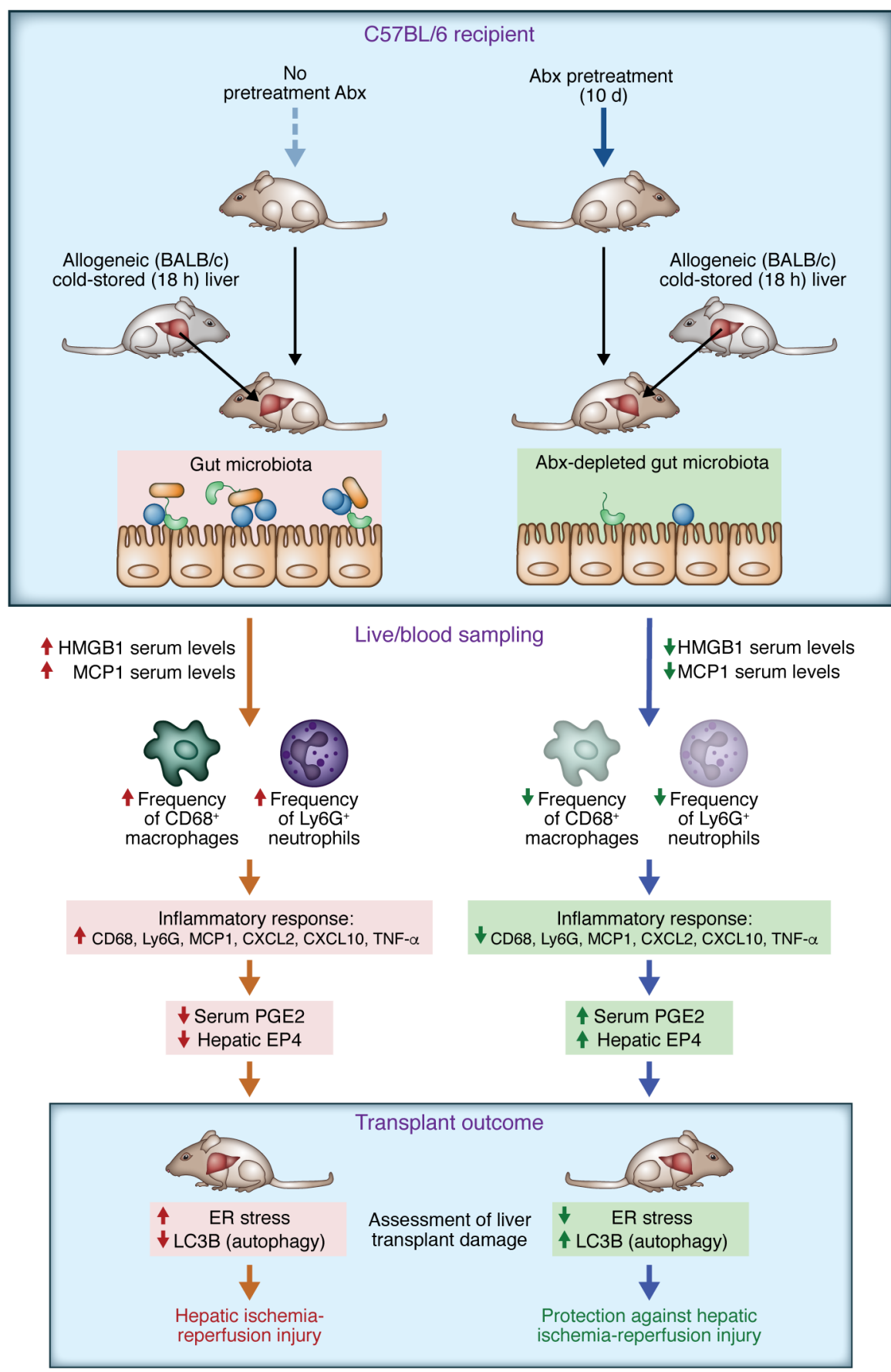

human transplant patients. To enhance the relevance of their findings, Nakamura et al. compared liver biopsies from transplant recipients who had received antibiotic treatment prior to surgery with those with limited antibiotic exposure (3). They found that recipients with extended antibiotic treatment had higher hepatic EP4 expression, decreased markers of ER stress, enhanced markers for autophagy, and decreased transaminitis. Subgroup analyses suggested that extended treat- ment with rifaximin (RFX), a minimally absorbed oral antimicrobial agent with broad activity within the gut, prevented IRI in human clinical liver transplantation.

Although it is clear from the results presented that the gut microbiota has an active role in determining IRI severity, a critical limitation of this study is that no microbiota characterization - either via "simple" taxa profiling using 16S rRNA gene sequencing or with more elaborate strategies such as metagenomic or
Figure 1. Antibiotic treatment prior to liver transplantation is protective against IRI. In this issue, Nakamura, Kageyama, Ito, Hirao, and colleagues show that the transplant recipient's microbiome influences graft function after liver transplantation. Liver transplantation in mice is accompanied by an increase in the microbemediated proinflammatory mediators HMGB1 and $\mathrm{MCP} 1$, which promote inflammatory responses in macrophages and neutrophils. The increase in proinflammatory immune cells is associated with decreased serum levels of PGE2 and reduced EP4 expression in the liver. EP4 antagonism in these animals increases the measures of IRI, including transaminitis, apoptosis, and hepatic and systemic inflammatory infiltrates and cytokines. Treatment of mice with antibiotics prior to allogeneic liver transplantation depletes the gut microbiota. This depletion results in a decrease in the inflammatory response and promotes antiinflammatory and homeostatic responses that lead to an increase in serum PGE2 and upregulation of hepatic EP4. Stimulation of EP4 enhances autophagy and suppresses ER stress and mTORC1 activity, thereby protecting against IRI. Abx, antibiotics. Illustrated by Rachel Davidowitz.

metatranscriptomic sequencing - was performed. The antibiotic used, amoxicillin, is a moderate-spectrum $\beta$-lactam antibiotic targeting both Gram-positive and Gram-negative bacteria that are likely to exert broad effects on the gut microbiota. Previous studies have shown that treatment with amoxicillin increases the amount of aerobic Gram-positive cocci and enterobacteria in the gut, among other potentially significant shifts (9). Because of the lack of microbiota characterization, Nakamura et al. were unable to assess which specific bacterial group(s) might be responsible for the effects on IRI severity and whether some bacterial groups might have a beneficial impact (3).

It is also unclear whether the effects exerted by the microbiota on Cox 2 gene regulation and PGE2 production were direct or indirect and what host cells and bacterial components were involved. Gut microbiota-mediated induction of the COX2 pathway, resulting in increased production of PGE2, has been previously demonstrated in obesity-associated liver cancer (9). In that study, it was shown that lipoteichoic acid, a Gram-positive gut microbial component, induced COX2 expression through a TLR2-mediated signaling pathway, facilitating tumor progression by suppressing antitumor immunity 
(9), consistent with the results presented in the current work (3).

Nakamura, Kageyama, Ito, Hirao, and colleagues interpreted their findings in antibiotic-treated liver transplant recipients to suggest that intervening on the microbiota in advance of transplantation prevented post-transplantation IRI (3). However, this should be qualified by several factors. First, the patients in the antibiotic-treated group were younger than those in the control group, which may be relevant, because younger patients typically have more robust immune responses than do older ones (10). Younger patients are also less likely to be frail (though this was not tested here), so the antibiotic recipients may have greater physiologic reserves and thus an improved ability to compensate during the transplantation surgery (11). Second, although an absence or limited use of antibiotics was associated with graft dysfunction by multivariable analysis, whether the organ was procured after cardiac death (rather than after brain death) was a greater determinant of graft dysfunction, suggesting that donor organ injury was a larger driver of recipient outcome. The authors did not explore whether an "at-risk" donor liver is somehow more susceptible to IRI in the context of altered recipient microbiota. Last, the patients who received antibiotics had higher model for end-stage liver disease (MELD) scores and were thus "sicker" than those who did not receive antibiotics. This is likely due to the direct association of infection and antibiotic requirement in patients with decompensated liver disease, thus increasing their MELD. This is an important consideration, because patients with decompensated cirrhosis have altered immune responses (12), which may have contributed to the observed changes in IRI after transplantation in this study.

\section{Clinical implications}

Clinically, the next obvious step is to ask how the antibiotic/microbiota axis contributes to an abrogated IRI response. Nakamura, Kageyama, Ito, Hirao, and colleagues did not characterize the effect of antibiotics on the microbiome of the human patients. This information is critical for a correct interpretation of their results, as the effects of antibiotic treatment on the gut microbiome can be quite variable $(13,14)$. Further, the authors could not precisely analyze all the individual antibiotics used in the transplant patients and the associated effects of these antibiotics on recipient outcomes or microbiome structure. Taken together, it is difficult to conclude that an altered microbiota necessarily accounted for differences in IRI. Rather, the authors found that antibiotic use prior to liver transplantation informs short-term outcomes (long-term results were not assessed). Additional work needs to be done to tease out exactly why this may be the case.

The results might also seem contradictory to the generally accepted concept, borne from human microbiome studies over the past ten-plus years, that dramatic disruptions of the microbiota, such as those induced by antibiotic treatments, tend to have a negative impact on overall health. For example, the microbiota acts as a barrier against colonization and overgrowth of potentially pathogenic and opportunistic microorganisms in the gut $(15,16)$, and its disruption following antibiotic therapy has been associated with complications such as recurring Clostridium difficile infections (16). Gut microbiota dysbiosis has also been shown to be a major contributor to inflammatory bowel diseases, cardiovascular diseases, and even autism and Parkinson's disease $(17,18)$. Previous studies have also suggested that antibiotic treatments can have long-lasting consequences, not only because of the risk of emergence and spread of antibiotic-resistant bacterial strains $(19,20)$, but also because microbiome dysbiosis can persist long after the treatment ends (13). Because the consequences of such permanent disruptions remain unknown, it will be important to follow this study with more in-depth analysis of the microbiota shifts in the treated transplant patients and identify the specific players and host factors involved in this response in order to potentially design a microbiota-based therapy for IRI that does not trigger unintended side effects.

\section{Acknowledgments}

The authors are supported by NIH grants 1R01AI062765, 1R01AI14496, 1R01EB026896, U01DK116095, 1R01AI123342, and U01 AIO63594 (to JSB) and 1R01AI143681 and 1R01HL148672 (to JSB and MFM).
Address correspondence to: Jonathan S. Bromberg, 22 South Greene St., S8B06; Baltimore, MD 21201, USA. Phone: 410.328.0008; Email: jbromberg@som. umaryland.edu.

1. Dutkowski P, Linecker M, DeOliveira ML, Müllhaupt B, Clavien PA. Challenges to liver transplantation and strategies to improve outcomes. Gastroenterology. 2015;148(2):307-323.

2. Zhai Y, Petrowsky H, Hong JC, Busuttil RW, Kupiec-Weglinski JW. Ischaemia-reperfusion injury in liver transplantation--from bench to bedside. Nat Rev Gastroenterol Hepatol. 2013;10(2):79-89.

3. Nakamura K, et al. Antibiotic pretreatment alleviates liver transplant damage in mice and humans. J Clin Invest. 2019;129(8):3420-3434.

4. Kolodziejczyk AA, Zheng D, Shibolet O, Elinav E. The role of the microbiome in NAFLD and NASH. EMBO Mol Med. 2019;11(2):e9302.

5. Blander JM, Longman RS, Iliev ID, Sonnenberg GF, Artis D. Regulation of inflammation by microbiota interactions with the host. Nat Immunol. 2017;18(8):851-860.

6. Zhou H, et al. The dichotomy of endoplasmic reticulum stress response in liver ischemia-reperfusion injury. Transplantation. 2016;100(2):365-372.

7. Zhu J, et al. Rapamycin protection of livers from ischemia and reperfusion injury is dependent on both autophagy induction and mammalian target of rapamycin complex 2-Akt activation. Transplantation. 2015;99(1):48-55.

8. Nakamura K, et al. Heme oxygenase-1 regulates sirtuin-1-autophagy pathway in liver transplantation: From mouse to human. Am J Transplant. 2018;18(5):1110-1121.

9. Loo TM, et al. Gut microbiota promotes obesityassociated liver cancer through PGE. Cancer Discov. 2017;7(5):522-538.

10. Wagner A, et al. Age-related differences in humoral and cellular immune responses after primary immunisation: indications for stratified vaccination schedules. Sci Rep. 2018;8(1):9825.

11. Busuttil RW, et al. Analysis of long-term outcomes of 3200 liver transplantations over two decades: a single-center experience. Ann Surg. 2005;241(6):905-916.

12. Albillos A, Lario M, Álvarez-Mon M. Cirrhosisassociated immune dysfunction: distinctive features and clinical relevance. J Hepatol. 2014;61(6):1385-1396.

13. Dethlefsen L, Relman DA. Incomplete recovery and individualized responses of the human distal gut microbiota to repeated antibiotic perturbation. Proc Natl Acad Sci U S A. 2011; 108(Suppl 1):4554-4561.

14. Zaura E, et al. Same exposure but two radically different responses to antibiotics: resilience of the salivary microbiome versus long-term microbial shifts in feces. MBio. 2015;6(6):e01693-e01615.

15. Pickard JM, Zeng MY, Caruso R, Núñez G. Gut microbiota: role in pathogen colonization, immune responses, and inflammatory disease. Immunol Rev. 2017;279(1):70-89. 
16. Kamada N, Chen GY, Inohara N, Núñez G. Control of pathogens and pathobionts by the gut microbiota. Nat Immunol. 2013;14(7):685-690.

17. Zhang YJ, Li S, Gan RY, Zhou T, Xu DP, Li HB. Impacts of gut bacteria on human health and diseases. Int J Mol Sci. 2015;16(4):7493-7519.

18. Durack J, Lynch SV. The gut microbiome: relationships with disease and opportunities for therapy. JExp Med. 2019;216(1):20-40.

19. Sullivan A, Edlund C, Nord CE. Effect of antimicrobial agents on the ecological bal- ance of human microflora. Lancet Infect Dis. 2001;1(2):101-114.

20. Jernberg C, Löfmark S, Edlund C, Jansson JK. Long-term impacts of antibiotic exposure on the human intestinal microbiota. Microbiology (Reading, Engl). 2010;156(Pt 11):3216-3223. 